\title{
Pegylated Liposomal Doxorubicin as a Chemotherapeutic Agent for Treatment of Canine Transmissible Venereal Tumor in Murine Models
}

\author{
Noa STETTNER ${ }^{1)}$, Ori BRENNER ${ }^{1)}$, Raya EILAM ${ }^{1)}$ and Alon HARMELIN ${ }^{1) *}$ \\ ${ }^{1)}$ Department of Veterinary Resources, Weizmann Institute of Science, P.O.B. 26, Rehovot, Israel 76100
}

(Received 24 February 2005/Accepted 13 July 2005)

\begin{abstract}
The effectiveness of Doxil as a new chemotherapeutic agent against canine transmissible venereal tumor was evaluated, using NOD/ SCID and CD1-nu xenograft mouse models and the response between the two mouse strains was compared. Samples of xenografted venereal tumor were inoculated SC into 20 six week-old NOD/SCID mice and 20 six week-old CD1-nu mice. Seven weeks later, tumor-bearing mice were divided into treatment and control groups. Treatment group was injected with Doxil (6 mg/kg, IP, as a single injection). Control group was injected with buffered saline $(0.75 \mathrm{cc}, \mathrm{IP})$. Tumor size was determined by caliper measurements and tumor response was assessed according to standard criteria. In both strains there was a significant decrease in tumor size in response to Doxil treatment $(\mathrm{P}<0.0001)$. In $\mathrm{CD} 1$-nu eight out of nine tumors $(88 \%)$ responded to the treatment, and in 2 cases complete remission was observed. In NOD/SCID group response to the treatment was seen in eight out of ten tumors (80\%) but none regressed fully. Response to the treatment was statistically equal in both strains even though the apoptotic rate, confirmed by TUNEL staining, was higher in NOD/SCID than in CD-1-nu (8.65\% and $0.7 \%$, respectively) and tumor infiltrating cells were different: eosinophils in NOD/SCID and CD45R-positive B lymphocytes, and plasma cells in CD-1-nu. In untreated CD1-nu mice, tumor progress was slower than in NOD/ SCID. Our results indicate that Doxil is effective against CTVT in mouse xenograft models.
\end{abstract} KEY WORDS: animal model, apoptosis, canine transmissible venereal tumor, chemotherapy, Doxil.

J. Vet. Med. Sci. 67(11): 1133-1139, 2005

Canine transmissible venereal tumor (CTVT), also known as canine transmissible sarcoma, is a naturally occurring contagious neoplasm of dogs located mainly on the external genitalia of both sexes $[2,21]$. It was defined as an undifferentiated round cell neoplasm probably of histiocytic origin $[19,21]$. The disease is mostly seen in free-roaming, sexually active dogs in tropical and subtropical climate [3]. The tumor can be transplanted as an allograft within the same species as well as to other canids such as foxes, coyotes, and jackals [2, 21]. Spontaneous and experimentally transplanted CTVT to an immunocompetent host shows an initial progressive growth, followed by a brief static period and then regression $[10,29]$. The occurrence of spontaneous tumor regression suggests that the immune response against the tumor plays a major role in determining the course of the disease [21].

Because spontaneous tumor regression is not observed in all cases, treatment is recommended [1]. Reported treatments of CTVT include surgical excision, radiotherapy, immunotherapy and chemotherapy. The last is considered the treatment of choice. Antimitotic agents such as vincristine, vinblastine and doxorobricin are the preferred chemotherapeutic agents [3]. Vincristine is the drug of choice. The use of doxorubricin is reserved for dogs that do not respond to vincristine, and is limited due to adverse reactions [1]. The toxicity of doxorobricin is characterized by dose limiting myelosuppression, stomatitis, alopecia, and significant risk of cardiotoxicity at cumulative doses.

\footnotetext{
* Correspondence to: Dr. Harmelin, A., Weizmann Institute of Science, Department of Veterinary Resources, P.O.B. 26 Rehovot, Israel 76100.
}

Pegylated liposomal doxorubicin $\left(\right.$ Doxil $^{\circledR}$ or Caelyx ${ }^{\mathrm{TM}}$ ) is a liposomal formulation of doxorubicin, believed to act via identical molecular mechanisms. The differences in its efficacy and toxicity are related to altered pharmacokinetics [28]. The drug has a long circulation time and a restricted volume of distribution as a result of the pegylated liposomes. In a number of tumor model studies, Doxil produced higher intratumoral drug concentrations and better therapeutic responses than equivalent doses of free doxorubicin $[4,5$, $8,27]$. In addition, its toxicity profile in dogs and humans is distinctly different from that of doxorubicin, and is characterized by dominant and dose limiting mucocutaneous toxicities (palmar-plantar erythrodysesthesia, and mucositis), but mild myelosupression and lower risk of cardiotoxicity [7]. Although the single maximum tolerated dose (MTD) of Doxil is lower than that of doxorubicin, the cumulative MTD dose of Doxil may be greater than that of free doxorubicin [7].

Doxil is currently approved for the treatment of AIDS related Kaposi's sarcoma [6]. Studies showed that Doxil is a highly potent and well-tolerated agent in this disease [7, 13]. CTVT and Kaposi's sarcoma might share some common characteristics: both neoplasms are sarcomas of uncertain origin, related to venereal transmission, and are more aggressive in immunosuppressed hosts $[2,14,26]$. We therefore queried whether Doxil may also be the treatment of choice against CTVT.

To facilitate studies of CTVT, a tumor xenograft model was established by transplantation of CTVT into NOD/ SCID mice [11, 12] and athymic nude mice - CD1-nu [16]. NOD/SCID strain is characterized by severe combined immunodeficiency. The nude strain lacks T cells activity. 
In the present study we investigated the effectiveness of Doxil against xenografted CTVT, using these two mouse strains. A secondary objective of this study was the evaluation of differences in tumor growth and response to treatment between the two strains, with particular attention to the role of tumor infiltrating cells and apoptosis.

\section{MATERIALS AND METHOD}

Animals: All experiments were preformed according to standard protocols [22] and were approved by the Weizmann Institutional Animal Care and Use Committee.

Two strains of mice were used in this study: NOD/LtSzscid (NOD/SCID) and CD1-nu. Both were supplied by the specific pathogen-free colony of the Department of Veterinary Resources of the Weizmann Institute of Science.

Preparation of cell suspension: Sample collection of CTVT and the initial cell transfer procedure into NOD/ SCID mice has been previously reported from this laboratory [12]. A xenografted transmissible venereal tumor (XTVT) was harvested and placed in cooled Hartmann's solution (Laboratoire Aguettan, Lyon, France). The tissue was minced with scissors to small fragments and gently homogenized mechanically through a disposable cell strainer. Cells were counted and suspended again in Hartmann's solution. A $0.2 \mathrm{~m} l$ of CTVT cell suspension containing $6 \times 10^{6}$ cells was injected SC into 20 six-week-old NOD/SCID and 20 six-week-old CD1-nu mice.

Chemotherapy: Seven weeks after injection, tumor size was determined by caliper measurements of length, width and depth. Tumor volume $\left(\mathrm{mm}^{3}\right)$ was calculated using the formula: length $\times$ width $\times$ depth $\times 0.5236$ [25]. Mice bearing a tumor were divided into treatment and control groups. Each group consisted of eight to ten mice. Treatment group was injected with Pegylated Liposomal Doxorubicin (Doxil $^{\circledR}$ ALZA Corp. Mauntain View, CA.) at a dose of 6 $\mathrm{mg} / \mathrm{kg}$, IP, as a single injection [28]. Control group was injected with buffered saline IP, as a single injection. Ciprofloxacin (Bayer AG, Leverkusen, Germany), $200 \mathrm{mg}$ in 100 $\mathrm{m} l$ water, was added to the drinking water of all groups for a week following treatment. Mice were monitored daily for development of any adverse reactions in response to the treatment by clinical observation. Tumors were measured twice a week. The mice were observed until tumors began to ulcerate or until tumor size reached an average diameter of $1.5 \mathrm{~cm}$. Mice were euthanatized with carbon dioxide.

Response criteria: Disappearance of all measurable tumors was considered as complete remission. A decrease in total tumor volume by $\geq 50 \%$ was defined as partial remission provided no new lesions developed and no progression of any existing lesions occurred. A decrease of $<50 \%$ in tumor volume and an increase of $\leq 25 \%$ in any of the measurable lesions were considered as no change. Progressive disease was defined as a $\geq 25 \%$ increase in the size of one or more lesions, or appearance of new lesions. Response rate was defined as the percentage of mice with complete remission and partial remission.
Statistical analysis: Results in the text and charts were reported as the average \pm standard error of the average. A two factors experiment with repeated measures on one of the factors test was used to determine statistical differences between treatment and control groups. Differences were considered statistically significant at $\mathrm{P} \leq 0.05$. Results were analyzed in square root of the volume because the assumption of homogeneity of variances was not met. One way ANOVA analysis of variance was used to determine the differences between the mice strains. T-test was used to determine differences in cell count and Mann-Whitney test was used to determine differences in apoptosis rate.

Histologic evaluation: Samples were fixed in neutral buffered $10 \%$ formalin, processed routinely, embedded in paraffin, sectioned at 4-5 $\mu \mathrm{m}$ and stained with haematoxylin and eosin.

Immunohistochemistry: Immunohistochemical detection of CD45R antigen was performed on sections of formalinfixed, paraffin embedded blocks using an immunoperoxidase complex procedure with diaminobenzidine as the chromogen. The primary antibody against CD45R (biotinconjugated rat anti-mouse CD45R/B220, PharMingen, San Diego, CA) was used at 1:100 dilution with a Vector M.O.M Kit (Vetor Laboratories, Burlingame, CA). Before staining slides were microwave treated for antigen exposure.

TUNEL staining for apoptotic cells: Specimens were fixed in neutral buffered $10 \%$ formalin for $24 \mathrm{hr}$, processed routinely, embedded in paraffin, sectioned at $7 \mu \mathrm{m}$ and collected on Super Frost slides (Menzel-Glaser, Frieburg, Germany). DNA fragmentation was determined by terminal deoxynucleotidyl transferase-mediated digoxigenin-dUTP nick-end labeling (TUNEL). Following deparaffinization and rehydration, the samples were washed with PBS, and preincubated with $0.5 \%$ triton $\mathrm{X}-100$ in PBS for $1 \mathrm{hr}$. The tissue was incubated with terminal deoxynucleotidyl transferase and digoxigenin-dUTP, and stained with fluorescinconjugated anti-digoxigenin antibody (ApopTag-Fluorescein in situ apoptosis detection kit, Intergen, N.Y., U.S.A.) following by Hoechst (Molecular Probs) counterstaining $(1: 2,000)$. Cover slips were mounted on a slide using gelmount solution (Polyscience, U.S.A.).

For quantitative analysis the number of TUNEL positive staining cells was counted and their density was expressed as the percentage of total number of Hoechst positive cells in $0.5 \mu \mathrm{m}^{2}$ area of 10 fields chosen randomly, using the Image-Pro plus $4.1^{\circledR}$ software (Media cybernetics, MD, U.S.A.).

\section{RESULTS}

The following results are based on evaluation of 19 tumors in NOD/SCID mice (ten in treatment and nine in the control group) and 17 tumors in CD1-nu mice (nine in treatment and eight in the control group). One mouse in NOD/ SCID group and three mice in CD1-nu group did not develop a detectable tumor at the injection site and were excluded. 
A

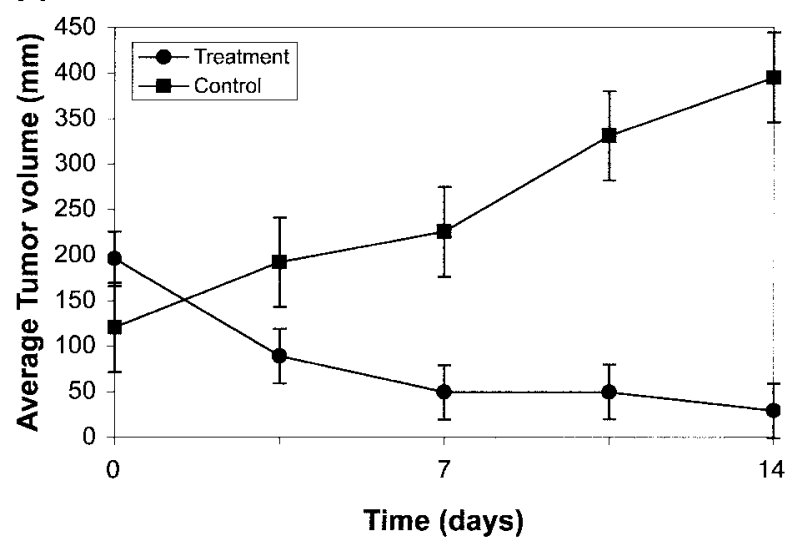

$B$

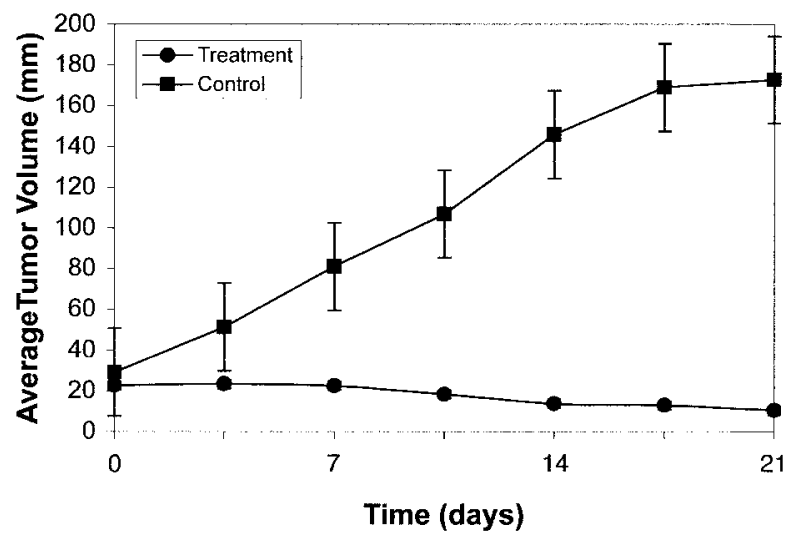

Fig. 1. Average volume of xenograft transmissible venereal tumor over time, transplanted in NOD/SCID (A) and CD1-nu mice (B), in response to Doxil treatment $(6 \mathrm{mg} / \mathrm{kg}$, IP, as a single injection). A significant reduction in tumor size was seen in both groups after the treatment $(\mathrm{P}<0.0001)$. Bars indicate the standard error from the average.
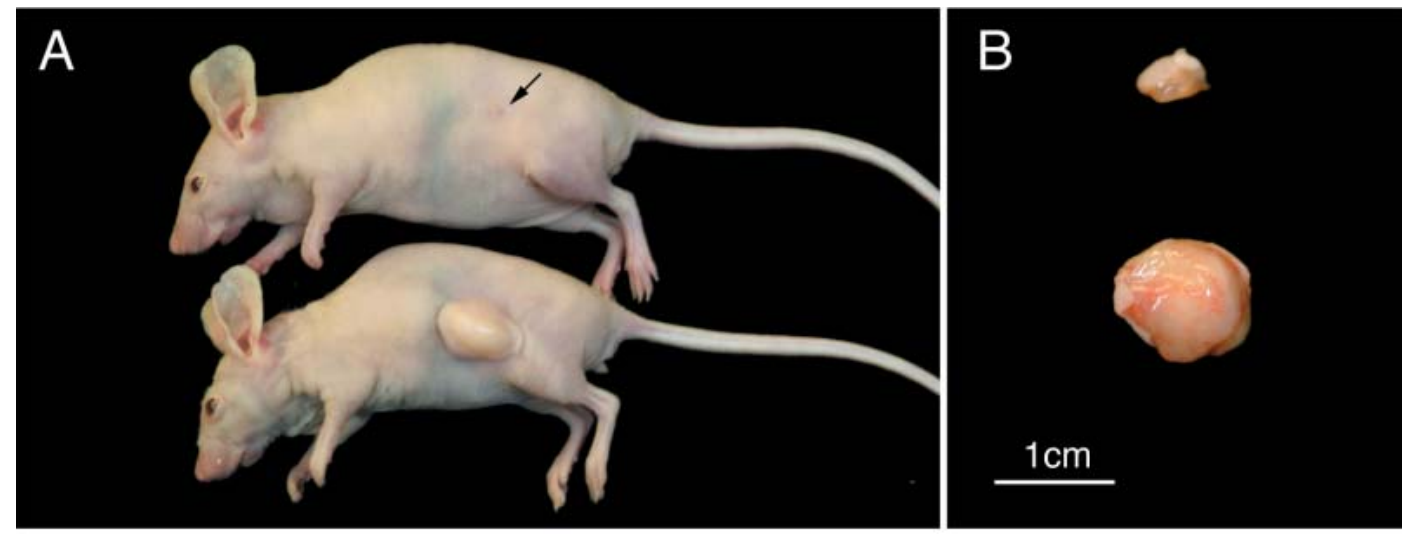

Fig. 2. (A) Gross appearance of NOD/SCID mice. Note markedly reduced tumor size in response to Doxil treatment (top) compared with control (bottom). Arrow indicates the location of the tumor. (B) The same tumors following excision. $\mathrm{Bar}=1 \mathrm{~cm}$.

Average tumor size in NOD/SCID mice control group was compared to that in CD1-nu mice control group in five measurements. Statistical analysis showed that tumors in untreated NOD/SCID mice developed faster compared with tumors in CD1-nu mice $(\mathrm{P}<0.05)$.

Response to treatment: Doxil at a dose of $6 \mathrm{mg} / \mathrm{kg}$ was well tolerated by the mice. There were no therapy-related deaths and none of the mice experienced overt adverse effects, as expected [7]. The changes of the average tumor size over time after Doxil treatment in NOD/SCID and CD1-nu mice are plotted in Fig. 1.

A significant reduction in tumor size was seen in both groups after Doxil treatment. Statistical analysis showed significant interaction between treatment and control groups $(\mathrm{P}<0.0001)$. In treatment groups tumor size decreased over time, while in control groups tumor size increased over time and the difference between the groups was significant. Figure 2 shows gross appearance of the tumor in NOD/SCID mice before and after the treatment. In CD1-nu eight out of nine tumors $(88 \%)$ responded to the treatment, with two showing complete remission $(22.2 \%)$. One tumor progressed. In the NOD/SCID group eight out of ten tumors $(80 \%)$ showed partial remission after the treatment but none had a complete remission. One tumor remained static and one progressed. Statistically, there was no significant difference in tumor regression following the treatment between CD1-nu and NOD/SCID mice.

\section{Histologic findings:}

Untreated control

XTVT in control groups, both NOD/SCID and CD-1-nu, had similar histologic features. The tumor was unencapsulated, well-circumscribed but infiltrative and composed of cohesive and densely cellular sheets of a uniform population of intermediate to large round cells interlaced by a delicate vascular stroma. Neoplastic cells had a moderate amount of frequently vacuolated eosinophilic cytoplasm with indistinct cytoplasmic margins. Nuclei were central, round, and large with fine to coarse granular chromatin and often a sin- 

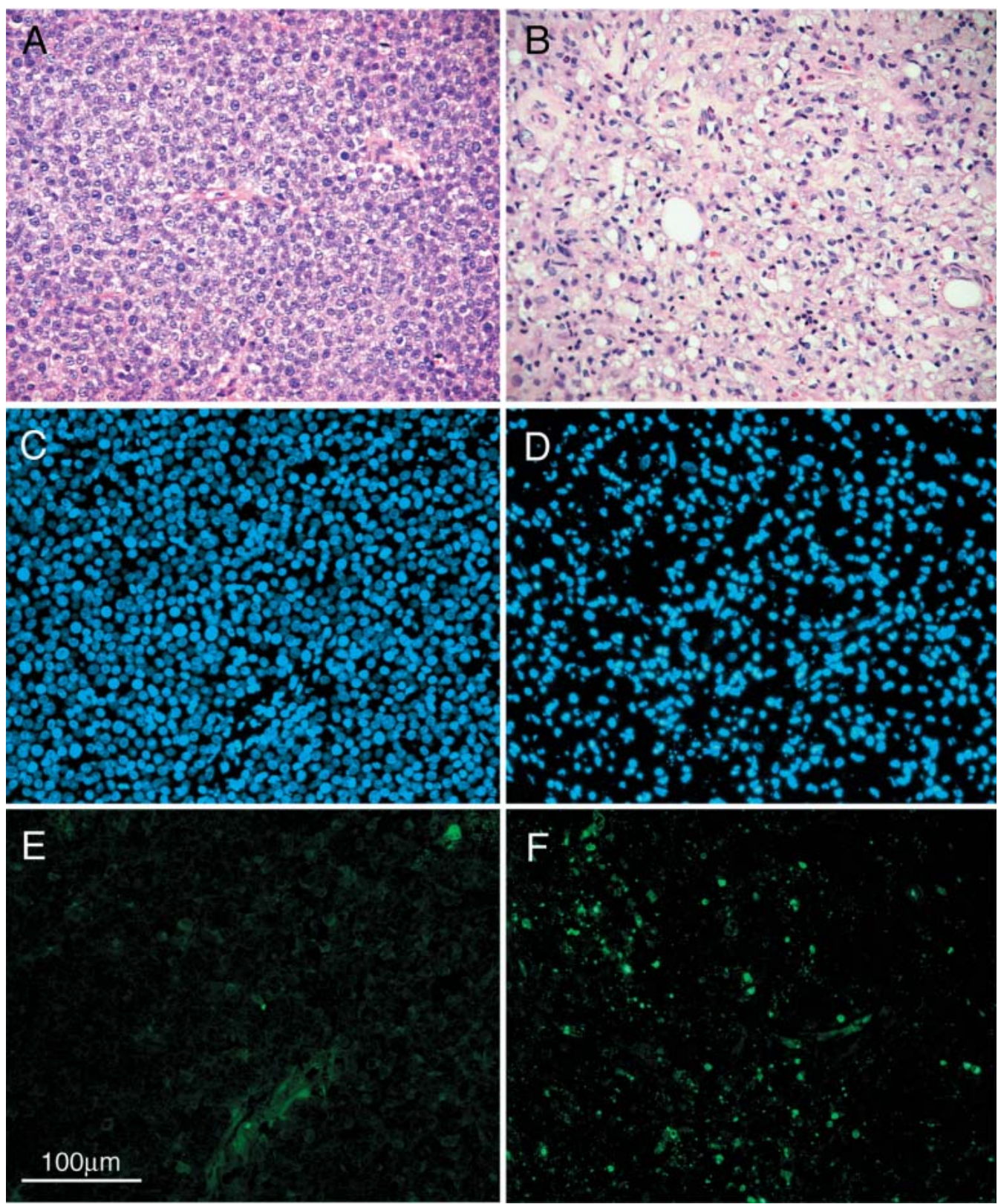

Fig. 3. The effect of Doxil on tumor regression in NOD/SCID mice. Representative sections are shown in pairs with controls on the left and treated on the right. (A and B) In the treated neoplasm cellular density is reduced and the cells are degenerate and necrotic. H\&E stain. (C and D) Total nuclear staining highlights the markedly reduced cellularity in the treated group. Hoechest stain. (E and F) Increased apoptosis in the treated group. Very few apoptotic cells are present in the control group. TUNEL stain. Bar=50 $\mu \mathrm{m}$.
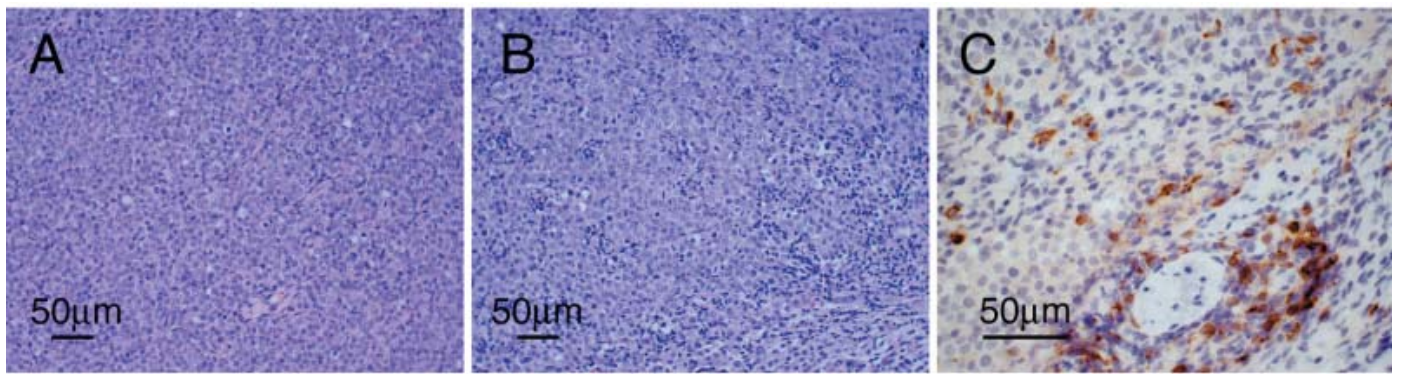

Fig. 4. Light microscopic features of XTVT in CD1-nu mice. (A) No appreciable inflammatory infiltration is seen in the control group. H\&E stain. (B) Multifocal lymphocytic infiltration following Doxil treatment. H\&E stain. (C) Immunohistochemical staining with CD45R antibody demonstrates many positive stained B lymphocytes in the treated group. Bar=50 $\mu \mathrm{m}$. 
gle magenta nucleolus. There was mild anisocytosis and anisokaryosis. Mitotic figures were common. Multifocal necrosis of variable but usually limited extent was observed. There was minimal inflammatory infiltration at the grafthost interface (Figs. 3A, 4A).

Treated NOD/SCID

The tumors were very small and partially surrounded by a thin fibrous capsule. Residual neoplastic cells were arranged in loose sheets and were degenerated with moderate to abundant and markedly vacuolated cytoplasm. Nuclei of viable neoplastic cells either retained their normal morphologic appearance as seen in control mice or appeared collapsed and distorted (Fig. 3B). Apoptotic / necrotic cells were common. There was minimal to mild multifocal predominantly infiltration of eosinophils at the margin of the tumor and to a lesser degree within the mass. The eosinophils were accompanied by cells tentatively identified as macrophages, but these cells could not be reliably distinguished from degenerated tumor cells.

Treated CD-1 nude

The tumors were very small. In some areas, the morphology of the neoplastic cells resembled to that of the control. In other areas the cells were degenerate and distributed more widely spaced. In both areas, both at the periphery and throughout the mass, there was moderate multifocal lymphocytic infiltration with rare eosinophils (Fig. 4B). Again, presumptive macrophages were seen. In some cases there was a small subcutaneous area of fibrosis with minimal amount of mononuclear infiltration, being consistent with full regression.

Immunohistochemistry: CD45R staining was used to evaluate the presence of $\mathrm{B}$ cells in the tumor. Positive cells were detected, especially at the periphery of the tumor. In control group the number of CD45R positive cells was very low. In treated CD1-nu their number was higher than in control group but still overall low (Fig. 4C).

TUNEL staining for apoptotic cells:

\section{Cell count}

Hoechst staining showed a significant reduction in the number of tumor cells in the treated versus untreated tumors in both mouse strains (Fig. 3C, D). In NOD/SCID mice the average number of cells counted in a $0.5 \mathrm{~mm}^{2}$ area of 10 fields chosen randomly, was $2068 \pm 83$ in control group versus $753 \pm 162$ in treatment group $(\mathrm{p}<0.0001)$. In CD1-nu mice the average number of cells was $1894 \pm 79$ in control group versus $1380 \pm 140$ in treatment group $(\mathrm{p}<0.0002)$. Apoptosis

TUNEL staining showed a significant increase in apoptosis after chemotherapy in both mice strains (Fig. 3E, F) $(\mathrm{P}=0.0079)$. In NOD/SCID mice the average percentage of apoptotic cells was $8.65 \%$ in treatment group versus $0.15 \%$ in control group. In CD1-nu mice the average percentage of apoptotic cells was $0.70 \%$ in treatment group versus $0.21 \%$ in control group.

\section{DISCUSSION}

Canine transmissible venereal tumor may regress spontaneously in immunocompetent hosts. Metastatic disease may occur in puppies and immunosuppressed dogs [21]. In our study we used a tumor xenograft in immunodeficient mouse strains as a model for the condition in immunosupressed dogs. The advantage of a murine model for CTVT other than the low cost, reproducibility and similarity with the natural disease, is that it allowed us to perform the initial experiment without the need for maintaining allogenic transfer in dogs [12]. In this study we demonstrated the effectiveness of a new chemotherapeutic drug, Doxil, against CTVT. Response to the treatment in CD1-nu group was $88 \%$ with $22 \%$ complete remission and in NOD/SCID was $80 \%$, after one Doxil injection $(6 \mathrm{mg} / \mathrm{kg}$, IP). The common chemotherapeutic regimen to treat CTVT is Vincristin sulphate 0.5 $\mathrm{mg} / \mathrm{m}^{2} \mathrm{IV}$ at weekly intervals for 3-6 weeks. This treatment is reported to be effective, except when the tumor is in the eye or brain [1]. So far Doxorubicin $\left(30 \mathrm{mg} / \mathrm{m}^{2}, \mathrm{IV}\right.$, once every 3 weeks) has been used in cases that had failed to respond to vincristine. However, the use of Doxorubicin is limited due to many adverse effects of the drug including dose limiting myelosuppression and cardiotoxicity. In a number of tumor models studied, Doxil was more effective than the same dose of Doxorubricin in inhibiting or halting tumor growth, in effecting cures and in prolonging survival of tumor bearing animals $[4,8,27,28]$. Toxicity observed in the animals following Doxil administration was less severe than that reported with Doxorubicin [28]. Doxilinduced skin toxicity is dose limiting in dogs, causing the single MTD to be lower than that of Doxorubicin. However, the cumulative dose of Doxil may be greater due to mild myelosuppressive effect and very low risk of cardiotoxicity [7].

A secondary objective was to study the difference in antitumor immunity against CTVT in NOD/SCID and CD1-nu mouse strains. The availability of various strains of mice with altered immunologic function makes murine models the most suitable choice for the study of immunologic mechanisms [12].

Untreated tumors grew faster in NOD/SCID than in CD1nu. The presence of active B lymphocytes and plasma cells only in CD1-nu mice might explain the slower growth of untreated tumors compared to tumor growth in NOD/SCID mice, which lack both $\mathrm{B}$ and $\mathrm{T}$ lymphocyte activity. These observations are compatible with other research comparing tumor growth in SCID and nude mice [23]. The important role played by peripheral B lymphocytes in the inhibition of TVT growth has been demonstrated [18].

Following the treatment with pegylated liposomal doxorubicin, a similar degree of tumor regression was seen in both strains. It has been suggested that anti-tumor immunity plays a role in tumor regression after modest chemotherapy [10]. Tumor regression is associated with tumor infiltrating lymphocytes, particularly $\mathrm{T}$ cells but also plasma cells and macrophages $[10,24]$. In CD1-nu mice, the proportion and 
the number of tumor infiltrating lymphocytes was higher in regressing than in proliferating tumors. This observation is in line with the results of other studies $[15,17,18,24]$. T cells are virtually absent in the mouse strains we used and therefore can not play a significant role in tumor regression in this system. In CD1-nu mice the dominant tumor infiltrating cells were B lymphocytes and plasma cells while in NOD/SCID they were eosinophils. As no significant difference in response to the treatment was observed between the strains, the contribution of these infiltrating cells to tumor regression remains to be determined.

An increase in apoptosis in both treatment groups was documented after chemotherapy both by TUNEL staining and conventional histologic examination. Increased apoptosis in regressing CTVT, either spontaneously or after modest chemotherapy has been reported [10]. Doxorubicin induces apoptosis in clinical concentrations [9]. Doxil contains doxorubicin as its active ingredient and is believed to act via the same mechanisms [28]. Thus it stands to reason that apoptosis in the tumor was induced by Doxil. Apoptosis in spontaneously regressing and treated TVT is considered to be associated with $T$ cell cytotoxicity $[17,21]$. In this study we observed apoptosis in the treatment groups in strains that lack $\mathrm{T}$ cell activity. Further studies are required to find out whether apoptosis was induced by different mechanisms or alternatively, the minimal residual activity of $\mathrm{T}$ cells in these strains was sufficient to induce it. Response to the treatment was statistically equal in both strains even though the apoptotic rate was higher in NOD/ SCID than in CD-1-nu ( $8.65 \%$ and $0.7 \%$, respectively). This would indicate that other mechanisms besides apoptosis are involved in Doxil-induced tumor regression. This is in line with current concepts regarding the mechanisms of action of anthracycline antibiotics which are the subject of considerable controversy [9]. At clinical drug concentration induction of DNA damage through interference with topoisomerase II and induction of apoptosis are thought to account for the antitumor effect of anthracyclin antibiotics $[9,21]$. The relevance of other mechanisms including inhibition of DNA synthesis, generation of free radicals, DNA adduction and formation of DNA cross linking, interference with DNA strand separation and DNA helicase and membrane mediated effects, remains questionable [9].

In this study, we used a xenotransplanted canine transmissible venereal tumor in mouse models to investigate the effectiveness of a new chemotherapeutic drug, Doxil. We demonstrated that Doxil is an effective treatment against this tumor. Although currently more expensive, it can provide a safer and probably a more effective alternative to doxorubicin. Studies in dogs are needed to validate these results on spontaneously developed tumors, to compare the response between Doxil and the currently used drugs- doxorubicin and vincristine, and to determine the optimal protocol. We anticipate that fewer treatments would be necessary compared to commonly used regimens. This would lower the overall treatment cost, reduce patient stress and increase client compliance.
The difference in tumor development between the mouse strains highlights the role of B lymphocytes and plasma cells in the course of the disease. In untreated mice, their presence may explain the slower tumor growth observed in CD1-nu than in NOD/SCID. In the treated mice, even though B lymphocytes and plasma cells were observed in CD1-nu and eosinophils in NOD/SCID, response to the treatment was equal. This would indicate that the role of each type of inflammatory cells infiltrated in the tumor tissue is either similar and/or not dominant in tumor regression after chemotherapy. We have shown that apoptosis is one of the mechanisms by which cell death occurred after the treatment in both strains.

Hopefully, these models could be used for further investigation of the role played by the immune system in CTVT development, and of the mechanisms by which cell death occurs in the regressing tumor.

\section{REFERENCES}

1. Aiello, S.E. 1998. Transmissible canine venereal tumor. pp. 683-684. In: The Merck Veterinary Manual. 8th ed., Merck \& Co., New Jersey.

2. Cohen, D. 1985. The canine transmissible venereal tumor: a unique result of tumor progression. Adv. Cancer Res. 43: 75112.

3. Das, U. and Das, A.K. 2000. Review of canine transmissible venereal sarcoma. Vet. Res. Commun. 24: 545-556.

4. Gabizon, A. and Martin, F. 1997. Polyethylene glycol-coated (pegylated) liposomal doxorubicin- Rationale for use in solid tumors. Drugs 54 (Suppl. 4): 15-21.

5. Gabizon, A., Isacson, R., Libson, E., Kaufman, B., Uziely, B., Catane, R., Ben-Dor, C.G., Rabello, E., Cass, Y., Peretz, T., Sulkes, A., Chisin, R. and Barenholz, Y. 1994. Clinical studies of liposome-encapsulated doxorubicin. Acta Oncol. 33: 779786.

6. Gabizon, A., Shmeeda, H. and Barenholz, Y. 2003. Pharmacokinetics of pegylated liposomal doxorubicin. Review of animal and human studies. Clin. Pharmacokinet. 42: 419-436.

7. Gabizon, A. 2001. Pegylated liposomal doxorubicin: Metamorphosis of an old drug into a new form of chemothetapy. Cancer Invest. 19: 424-436.

8. Gabizon, A.A., Pappo, O., Goren, D., Chemla, M., Tzemach, D. and Horowitz, A.T. 1993. Preclinical studies with doxorubicin encapsulated in polyethyleneglycol-coated liposomes. $J$. Liposome Res. 3: 517-528.

9. Gewirtz, D.A. 1999. A critical evaluation of the mechanisms of action proposed for the antitumor effects of the anthracycline antibiotics adriamycin and daunorubicin. Biochem. Pharmacol. 7: 727-741.

10. Gonzalez, C.M., Griffey, S.M., Naydan, D.K., Flores, E., Cepeda, R., Cattaneo, G. and Madewell, B.R. 2000. Canine transmissible venereal tumour: a morphological and immunohistochemical study of 11 tumours in growth phase and during regression after chemotherapy. J. Comp. Pathol. 122: 241-248.

11. Harmelin, A., Pinthus, J.H., Friedmann-Morvinski, D., Kaufman, K. and Brenner, O. 2002. Lack of MHC expression and retention of ultrastructural characteristics by xenograft transmissible venereal tumor cells in SCID mice Vet. Immunol. Immunopathol. 86: 245-249.

12. Harmelin, A., Pinthus, J.H., Katzir, N., Kapon, A., Volcani, Y., 
Amariglio, E.N. and Rehavi, G. 2001. Use of a murine xenograft model for canine transmissible venereal tumor. $\mathrm{Am}$. J. Vet. Res. 62: 907-911.

13. Harrison, M., Tomlinson, D. and Stewart, S. 1995. LiposomalEntrapped Doxorubicin: An active agent in AIDS-related Kaposi's sarcoma. J. Clin. Oncol. 13: 914-919.

14. Hayes, H.M. Jr, Biggar, R.J., Pickle, L.W. and Hoover, R. 1983. Canine transmissible venereal tumor: A model for Kaposi's sarcoma. Am. J. Epidemiol. 117: 108-109.

15. Hill, D.L., Yang, T.J. and Wachtel, A. 1984. Canine transmissible venereal sarcoma: Tumor cell and infiltrating leukocyte ultrastructure at different growth stages. Vet. Pathol. 21: 3945.

16. Holmes, J.M. 1981. Measurement of the rate of death of canine transmissible venereal tumour cells transplanted into dogs and nude mice. Res. Vet. Sci. 30: 248-250.

17. Hsiao, Y.W., Liao, K.W., Hung, S.W. and Chu, R.M. 2002. Effect of tumor infiltrating lymphocytes on the expretion of MHC molecules in canine transmissible venereal tumor cells. Vet. Immunol. Immunopathol. 87: 19-27.

18. Liano, K.W., Hung, S.W., Hsiao, Y.W., Bennett, M. and Chu, R.M. 2003. Canine transmissible venereal tumor cell depletion of B lymphocytes: molecule(s) specifically toxic for B cells. Vet. Immunol. Immunopathol. 92: 149-162.

19. Marchal, T., Chabanne, L., Kaplanski, C., Rigal, D. and Magnol, J.P. 1997. Immunophenotype of the canine transmissible venereal tumor. Vet. Immunol. Immunopathol. 57: 1-11.

20. Mizutani, H., Tada-Oikawa, S., Hiraku, Y., Kojima, M. and Kawanishi, S. 2005. Mechanism of apoptosis induced by doxorubicin through the generation of hydrogen peroxide. Life Sci. 76: 1439-1453.

21. Mukaratirwa, S. and Gruys, E. 2003. Canine transmissible venereal tumor: cytogenetic origin, immunophenotype, and immunobiology. A review. Vet. Q. 25: 101-111.

22. National Research Council. 1996. Guide for the Care and Use of Laboratory Animals. Washington, D.C. National Academy Press.

23. Nomura, T., Takahama, Y., Hongyo, T., Takatera, H., Inohara, H., Fukushima, H., Ono, S. and Hamaoka, T. 1991. Rapid growth and spontaneous metastasis of human germinal tumors ectopically transplanted into scid (severe combined immunodeficiency) and scid-nudestreaker mice. Jpn J. Cancer Res. 82: 701-709.

24. Perez, J., Day, M.J. and Mozos, E. 1998. Immunohistochemical study of the local inflammatory infiltrate in spontaneous canine transmissible venereal tumor at different stages of growth. Vet Immunol. Immunopathol. 64: 133-147.

25. Pinthus, J.H., Waks, T., Schindler, D.G., Harmelin, A., Said, J.W., Belldegrum, A., Ramon, J. and Eshhar, Z. 2000. WISHPC2: A unique xenograft model of human prostatic small cell carcinoma. Cancer Res. 60: 6563-6567.

26. Rechavi, G., Katzir, N. and Ramot, B. 1991. Kaposi's sarcoma among AIDS patients: Transmissible venereal tumor by cell engrafment? Med. Hypotheses 34: 338-341.

27. Vail, D.M., Kravis, L.D., Cooley, A.J., Chun, R. and MacEwen, E.G. 1997. Preclinical trial of doxorubicin entrapped in sterically stabilized liposomes in dogs with spontaneously arising malignant tumors. Cancer Chemother. Pharmacol. 39: 410-416.

28. Working, P.K. and Dayan, A.D. 1996. Pharmacological-toxicological expert report. CAELYX ${ }^{\mathrm{TM}}$. (Stealth ${ }^{\circledR}$ liposomal doxorubicin $\mathrm{HCl}$ ). Hum Exp. Toxicol. 15: 751-785.

29. Yang, T.J. and Jones, J.B. 1973. Canine transmissible venereal sarcoma. Transplantation studies in neonatal and adult dogs. $J$. Natl Cancer Inst. 51: 1915-1918. 